\title{
Quantitative High-Field Diffusion Tensor Imaging of Cerebral White Matter in Asymptomatic High-Grade Internal Carotid Artery Stenosis
}

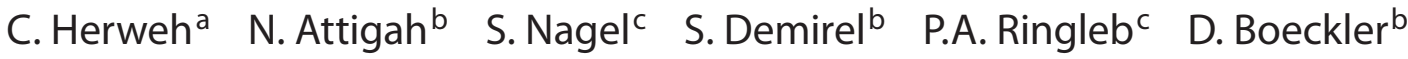 \\ M. Bendszus a

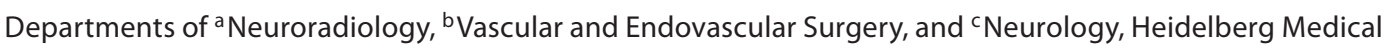 \\ School, University of Heidelberg, Heidelberg, Germany
}

\section{Key Words}

Cerebrovascular disease $\cdot$ Diffusion tensor imaging •

Neuroimaging

\begin{abstract}
Background: Recently, several studies using diffusion-sensitized MRI reported changes in patients with high-grade internal carotid artery stenosis (ICAS) suggestive of subtle brain tissue damage. We used diffusion tensor imaging (DTI) to investigate the microstructural cerebral white matter integrity in asymptomatic patients with high-grade ICAS. Methods: In 15 asymptomatic patients with unilateral high grade (>70\%) ICAS, we used 3T MRI including DTI. We used a region-of-interest approach comparing quantitative DTI metrics between both hemispheres including the so-called border zones. MR images were also assessed for periventricular white matter lesions (PWML) as well as collaterals via the circle of Willis. Results: There was no significant intraindividual difference of fractional anisotropy or mean diffusivity values between the hemispheres for any region. PWML was graded $0^{\circ}$ in 6 patients, $\mathrm{I}^{\circ}$ in 9 and $\mathrm{II}^{\circ}$ in 2. Conclusions: In clinically asymptomatic patients with high-grade unilateral ICAS, there was no difference between the DTI parameters of the affected and the unaffected hemisphere. These findings contrast with other studies in asymptomatic high-grade ICAS, which is likely due to patient selection. These findings
\end{abstract}

\section{KARGER \\ Fax +4161306 1234 \\ E-Mail karger@karger.ch}

www.karger.com
(C) 2012 S. Karger AG, Basel

0014-3022/12/0674-0246\$38.00/0

Accessible online at:

www.karger.com/ene argue against concomitant chronic tissue integrity changes and implicate the benignity of asymptomatic carotid artery disease in individual patients. Copyright $\Theta 2012$ S. Karger AG, Basel

\section{Introduction}

High-grade atherosclerotic narrowing of the extracranial vessels is a well-recognized cause of cerebral ischemia and can be treated in these cases, either by thromboendarterectomy or percutaneous transluminal angioplasty. In asymptomatic patients with stenosis of the internal carotid artery (ICAS), the indication for an intervention, whether surgical or endovascular, is controversial since these patients carry only a moderate annual risk of $2 \%$ to suffer from a disabling ischemic stroke $[1,2]$. An ongoing multicenter trial is comparing interventional methods with upto-date medical prophylaxis in the treatment of asymptomatic ICAS [3]. Whereas the aim of these different approaches is primary prophylaxis of ischemic cerebral stroke, several groups have reported an increase of diffusion of free water molecules in cerebral tissue of the dependent hemisphere downstream to an ICAS in symp-

C. Herweh and N. Attigah contributed equally to the manuscript. 
Table 1. Summary of epidemiological data, ultrasound and MRI findings as well as risk factors and comorbidities

\begin{tabular}{|c|c|c|c|c|c|c|c|c|c|c|c|c|c|}
\hline \multirow[t]{2}{*}{ Age } & \multirow[t]{2}{*}{ Sex } & \multirow[t]{2}{*}{ Side } & \multicolumn{2}{|c|}{ Stenosis, \% } & \multicolumn{2}{|c|}{$\begin{array}{l}\text { Intracranial } \\
\text { collaterals }\end{array}$} & \multirow[t]{2}{*}{ PWML } & \multicolumn{4}{|c|}{ Cardiovascular risk factors } & \multicolumn{2}{|c|}{$\begin{array}{l}\text { Cardiovascular } \\
\text { comorbidities }\end{array}$} \\
\hline & & & affected & unaffected & Acom & $\begin{array}{l}\text { ipsi. } \\
\text { Pcom }\end{array}$ & & aHT & $\begin{array}{l}\text { chol/ } \\
\text { lip }\end{array}$ & $\mathrm{DM}$ & nic & CAD & PAD \\
\hline 64 & M & left & 70 & 50 & & & 0 & yes & yes & no & yes & yes & no \\
\hline 83 & M & left & 85 & 50 & yes & yes & 1 & yes & no & no & no & no & no \\
\hline 78 & M & left & 80 & 50 & yes & no & 1 & no & no & no & no & no & no \\
\hline 69 & M & right & 90 & 50 & no & no & 1 & yes & no & yes & yes & yes & yes \\
\hline 61 & $\mathrm{~F}$ & left & 70 & 50 & yes & yes & 1 & yes & yes & no & no & no & no \\
\hline 63 & $\mathrm{~F}$ & left & 90 & 50 & no & no & 0 & yes & yes & yes & no & no & no \\
\hline 67 & $\mathrm{~F}$ & left & 75 & 50 & yes & no & 0 & yes & yes & no & yes & yes & no \\
\hline 63 & M & right & 80 & 60 & yes & yes & 0 & yes & yes & yes & yes & yes & yes \\
\hline 65 & $\mathrm{~F}$ & right & 80 & 50 & yes & no & 1 & yes & yes & no & no & no & no \\
\hline 69 & M & right & 85 & 50 & yes & no & 1 & yes & yes & no & yes & yes & no \\
\hline 69 & $\mathrm{~F}$ & left & 90 & 50 & no & no & 0 & yes & no & yes & no & no & no \\
\hline 62 & M & right & 90 & 50 & no & no & 1 & no & no & yes & no & no & no \\
\hline 65 & M & right & 70 & 50 & yes & no & 2 & yes & no & yes & no & yes & no \\
\hline 68 & $\mathrm{~F}$ & right & 85 & 55 & yes & no & 2 & yes & yes & no & no & yes & no \\
\hline 76 & $\mathrm{M}$ & right & 90 & 50 & yes & yes & 2 & yes & no & no & no & no & no \\
\hline
\end{tabular}

PWML are graded according to Fazekas et al. [11]. Acom = Anterior communicating artery; Pcom = posterior communicating artery; $\mathrm{aHT}$ = arterial hypertension; chol/lip = hypercholesterolemia/hyperlipidemia; $\mathrm{DM}=$ diabetes mellitus; nic = nicotine abuse; $\mathrm{CAD}=$ coronary artery disease $\mathrm{PAD}=$ peripheral artery disease.

tomatic as well as asymptomatic patients [4-6], which might indicate subtle tissue damage. The deleterious effect of chronic hypoperfusion on brain tissue has been postulated to be one explanation for this observation [7]. Diffusion tensor imaging (DTI) is a functional MR method that probes random motion of free water molecules, thereby allowing conclusions especially regarding brain white matter histoarchitecture. Among derived quantitative parameters, fractional anisotropy (FA) in particular is considered to be a reliable marker of white matter microstructural integrity [8]. FA is thought to reflect fiber density and myelination in white matter and is arbitrarily scaled between one and zero and describes the degree of anisotropy of a diffusion process; a value of zero means that diffusion is isotropic, i.e. it is equally restricted in all directions, otherwise a value of one means that diffusion occurs only along one axis and is fully restricted along all other directions. Another parameter often employed is mean diffusivity (MD), which corresponds to the apparent diffusion coefficient (ADC) as derived from diffusion-weighted imaging (DWI). DTI has been shown to be a reliable method for the quantification of white matter integrity; furthermore, advantage can be derived from high magnetic field strengths [9]. In order to prove and further describe pos- sible changes ipsilateral to an ICAS in asymptomatic patients, we used quantitative DTI to investigate cerebral white matter in different regions including the so-called vascular border zones where symptomatic ischemia typically occurs [10] by a region-of-interest (ROI) analysis.

\section{Materials and Methods}

\section{Patients}

We recruited 22 consecutive patients who were referred either to the department of vascular and endovascular surgery, neurology or neuroradiology due to a recently diagnosed high-grade $(>70 \%)$ unilateral ICAS detected in the framework of routine ultrasound examinations prompted by concomitant coronary or peripheral artery disease, or due to an existing risk factor profile. Patients were included only if they reported no previous specific cerebral or ocular symptoms and if neurological examination performed by a neurologist was unremarkable. In all patients, physical and neurological examination (including Barthel score), assessment of cardiovascular risk factors and extracranial Doppler/duplex sonography was performed. All patients underwent an MR examination at 3 T including DWI, FLAIR, MR angiography and DTI sequences. Patients were not included if MRI showed signs of prior embolic infarction ipsilateral and thus attributable to ICAS. Written informed consent was obtained from all patients and the study was approved by the local ethics committee. 
Fig. 1. ROIs for assessment of quantitative FA and ADC values. Displayed are exemplary ROIs for different white matter regions in the left hemisphere in border zones: parasagittal frontal (a), frontal (b) and parietal (c) with periventricular (1) and subcortical (2) locations, respectively, as well as in the central subcortical white matter (d) and in the posterior limb of the internal capsule (e).
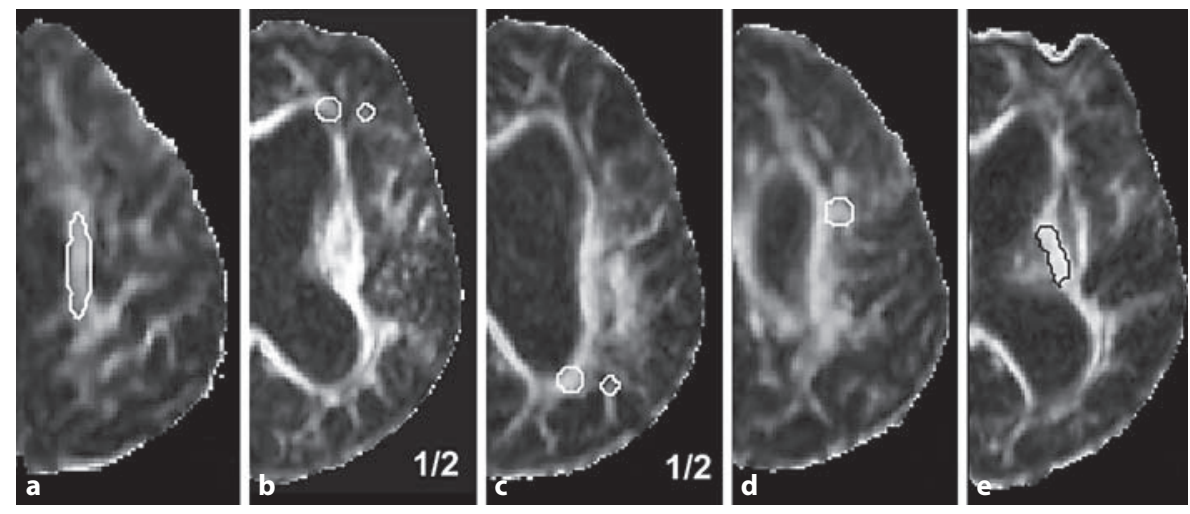

\section{Ultrasound}

Obstructive lesions of the internal carotid artery were diagnosed by noninvasive carotid studies using continuous-wave Doppler and duplex sonography with frequency and power-based color coding (7.5-MHz linear probe, Xario; Toshiba Medical, Tustin, Calif., USA). Stenosis of the proximal internal carotid artery was classified as a high-grade lesion if there was a diameter reduction of $>70 \%$ in duplex imaging with a strong local flow increase $(>230 \mathrm{~cm} / \mathrm{s})$ in the duplex examination and poststenotic decrease of systolic flow velocity in the continuous-wave Doppler study. Demographic data and extracranial Doppler findings are summarized in table 1.

\section{MRI Protocol}

MRI was performed on a 3T MR unit (Trio; Siemens Medical, Erlangen, Germany) using a 12-channel head coil. The protocol comprised a FLAIR sequence (TE $97 \mathrm{~ms}$, TR 10,000 ms, TI 2,500 ms, PAT 2, FOV $220 \mathrm{~mm}$, matrix $256 \times 192$, slice thickness $6 \mathrm{~mm}$ ), time-of-flight angiography (TE $3.73 \mathrm{~ms}$, TR $22 \mathrm{~ms}$, PAT 2, flip angle $18^{\circ}$, FOV $200 \mathrm{~mm}$, matrix $384 \times 256$, slice thickness $0.64 \mathrm{~mm}$ ) and DWI (TE $86 \mathrm{~ms}$, TR 8, $400 \mathrm{~ms}$, PAT 2, FOV $256 \mathrm{~mm}$, matrix $128 \times 128$, slice thickness $6 \mathrm{~mm}$ ) in 6 noncollinear directions with a b value of 0 and 1,000 with 5 averages to increase the signal-to-noise ratio.

\section{Image Analysis}

FLAIR and diffusion-weighted images were analyzed to rule out any nonischemic as well as chronic or silent acute ischemic lesions. Severity of periventricular white matter lesions (PWML) on FLAIR images was rated using a modified version of the visual scale of Fazekas et al. [11], ranging from 0 to 3, that scores deep and subcortical white matter lesions in three categories of mild $\left(1^{\circ}\right)$, moderate $\left(2^{\circ}\right)$, and severe $\left(3^{\circ}\right)$ white matter lesions. In an attempt to estimate the degree of intracranial collateralization the patency of the anterior and posterior communicating artery on MRA was recorded.

Diffusion-weighted images were transferred to a standard PC (1.8 GHz, 768 MB RAM). The 'brain extraction tool' (Oxford Centre for Functional Magnetic Resonance of the Brain, Oxford University, Oxford, UK, http://www.fmrib.ox.ac.uk/analysis/research/bet/) was used to remove extracerebral tissue (e.g. skull, meninges, eyes, etc.) from the images. In order to correct for arti- facts due to patient motion and eddy currents, the images were realigned employing the AIR software described in detail elsewhere [12]. For better anatomical orientation the images were interpolated post-hoc to yield a $256 \times 256$ matrix. The diffusion tensor was calculated for each voxel, diagonalized to yield eigenvectors and values of FA and MD. We used the method described by Basser et al. [13] and a customized MatLab ${ }^{\mathrm{TM}}$-based in-facility software. To assess quantitative FA and MD values, we performed an ROI analysis. ROIs were placed in white matter in regions considered to be hemodynamic risk zones (so-called border zones) between the territories of the middle, anterior and posterior cerebral arteries: parasagittal within the frontal lobe as well as in the periventricular and subcortical white matter independently in the frontal and parietal region. ROI placement in the subcortical white matter was chosen to avoid any PWML. Additional ROIs were placed in the subcortical white matter of the central region and in the posterior limb of the internal capsule to yield internal reference values. See figure 1 for graphical illustration of exemplary ROIs.

\section{Statistical Analysis}

For statistical analysis we used a standard software package (SPSS 17 ${ }^{\mathrm{TM}}$, SPSS Inc., Chicago, Ill., USA). The KolmogorovSmirnov test was used to check for normal distribution. To compare means between both hemispheres, we used the paired-samples $t$ test.

\section{Results}

Fifteen patients were included in the final analysis: 6 were female, with a median age of 68 years. Seven patients had to be excluded: 2 patients exhibited only moderate stenosis on either side, 1 patient became symptomatic prior to the MR examination, 1 patient had bilateral highgrade ICAS, 1 patient had developed an occlusion at the time of MR examination and 2 patients had signs of symptomatic chronic infarction. The target stenosis was on the left side in 8 cases and the median stenosis grade was $85 \%$ (range: $70-90$ ), while the contralateral side had 

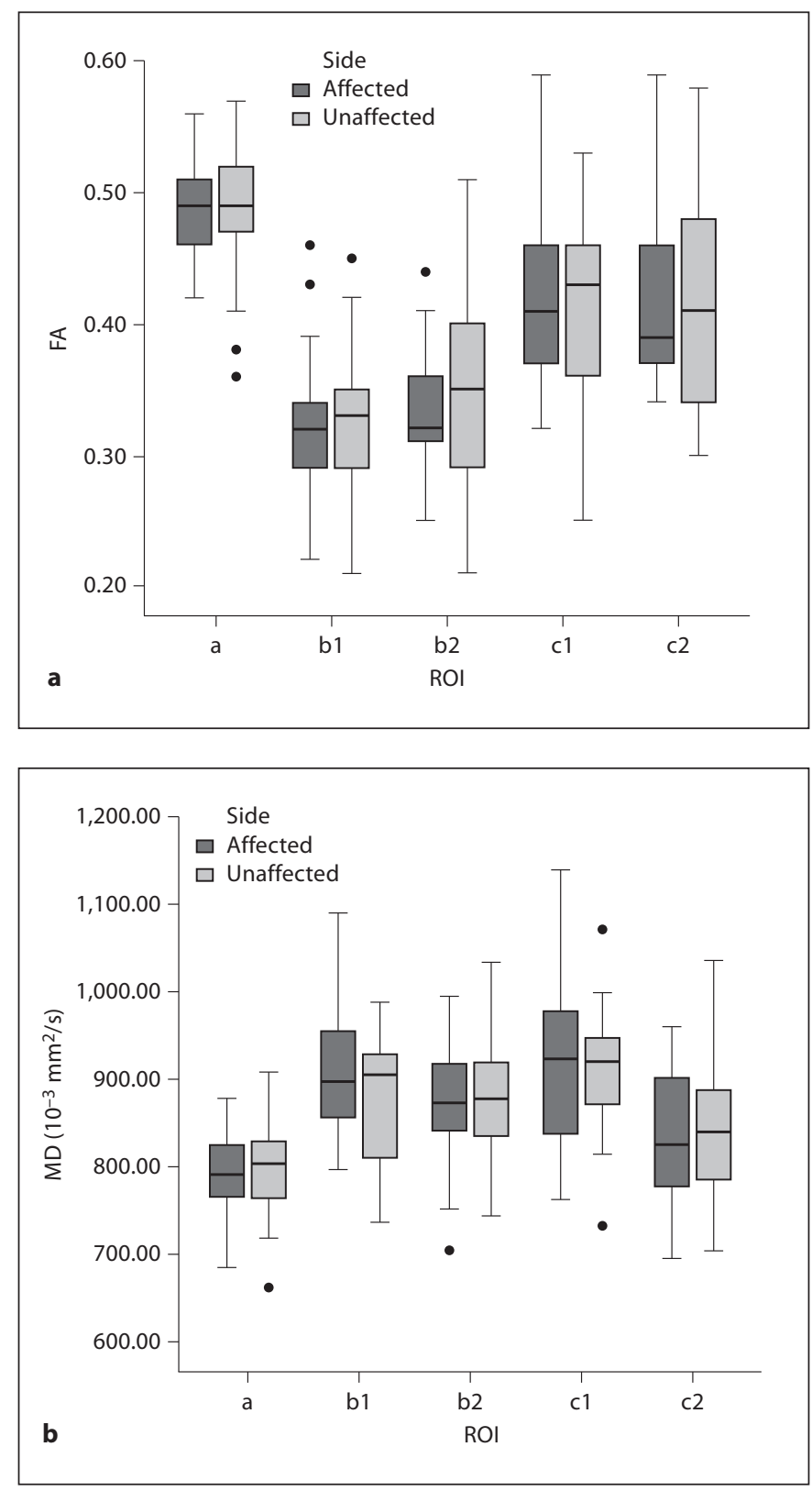

Fig. 2. Box plots illustrating quantitative FA (a) and $M D(\mathbf{b})$ values for ROIs in both hemispheres. For anatomical location of the ROIs please refer to the legend of figure 1.

a median stenosis grade of 50\% (range: 50-60) as measured with ultrasound. There were signs of prior small embolic infarction in a cortical/subcortical location in the contralateral hemisphere in 1 patient. PWML was grade 1 in 9 patients and grade 2 in 3 patients. MR angiography showed both the anterior and ipsilateral posterior communicating artery in 4 patients, one communi-
Table 2. Quantitative FA and MD values

\begin{tabular}{llll}
\hline ROI locations & Hemispheres & $\mathrm{p}$ \\
\cline { 2 - 3 } & affected & unaffected & \\
\hline
\end{tabular}

FA values

Frontal border zone

Periventricular

Subcortical

$0.32 \pm 0.07$

$0.33 \pm 0.06$

0.68

Parietal border zone

Periventricular

$0.34 \pm 0.05$

$0.35 \pm 0.08$

0.88

Subcortical

$0.42 \pm 0.07 \quad 0.41 \pm 0.08 \quad 0.4$

Parasagittal

$0.41 \pm 0.07$

$0.41 \pm 0.09$

0.71

Central subcortical

$0.49 \pm 0.04$

$0.48 \pm 0.06$

0.29

PLIC

$0.6 \pm 0.05$

$0.59 \pm 0.05$

MD values

Frontal border zone

$\begin{array}{llll}\text { Periventricular } & 921.84 \pm 89.64 & 877.08 \pm 78.27 & 0.67\end{array}$

Subcortical

$862.34 \pm 75.61 \quad 866.54 \pm 72.29 \quad 0.87$

Parietal border zone

Periventricular

Subcortical

$921.75 \pm 81.31 \quad 902.26 \pm 62.25 \quad 0.39$

Parasagitta

$825.80 \pm 8$

$841.74+85.81$

0.71

Central subcortical

$787.19 \pm 54.98 \quad 792.58 \pm 62.21 \quad 0.29$

PLIC

$787.97 \pm 66.28$

$851.62 \pm 83.81$

0.77

$\mathrm{MD}$ values are $10^{-3} \mathrm{~mm}^{2} / \mathrm{s}$; arithmetic mean $\pm \mathrm{SD}$. PLIC $=$ Posterior limb of the internal capsule.

cating artery in 7 patients, and none in 6 patients. Hypertension was the most frequent cardiovascular risk factor and present in 13 patients. Seven patients had known coronary arterial disease of which 2 also had peripheral artery disease (for a detailed summary of clinical and demographic data, risk factors and comorbidities see table 1). In an intraindividual comparison between the ipsilateral and contralateral hemisphere there were no significant differences of FA or MD values for any region. See table 2 for quantitative results and $p$ values, and figure 2 for a graphical illustration.

\section{Discussion}

In the present group of 15 patients with unilateral high-grade ICAS, we found no significant difference of quantitative DTI parameters between the ipsi- and contralateral hemisphere, neither in the watershed regions of the middle cerebral artery territory nor in the other regions analyzed. 
To date, only a few studies have assessed the integrity of white matter in patients with carotid artery stenosis using diffusion-sensitive MRI. These studies vary significantly in terms of study design and patient selection. Inoue et al. [4] compared FA values from a ROI-based analysis in the centrum semiovale of both hemispheres in 30 symptomatic patients and found reduced values on the affected side. In this study, no analysis of pre-existing white matter lesions was performed and no information concerning the neurological status of the patients is given. Soinne et al. [6] evaluated ADC values using DWI in symptomatic as well as asymptomatic patients with unilateral high-grade ICAS in comparison to healthy controls. They showed that the ADC values in the ipsilateral white matter and the watershed regions not affected by leukoaraiosis were higher than contralateral and higher than in control subjects. Interestingly, they reported no differences between the symptomatic and asymptomatic patient groups. Moreover, they found a slightly larger leukoaraiotic area in the ipsilateral hemisphere of the patients and argued that this might be the consequence of carotid disease and that the elevated ADC values might represent a 'preleukoaraiosis'. According to this study, we assessed quantitative values within watershed regions by ROIs in the periventricular as well as in the subcortical white matter. Whereas leukoaraiosis is typically encountered in the first, it could be avoided in the latter location. Therefore, the present findings are independent of PWML. In contrast to Soinne et al. [6] the prevalence of PWML was rather small in the present study. Shiraishi et al. [5] used DTI with low (fast) and high (slow) b values to study FA and MD values in the internal capsule, the corona radiata and the centrum semiovale in 6 patients with high-grade stenosis or occlusion of either the internal carotid artery or middle cerebral artery who had not shown symptoms during the past 3 months. The authors report that the slow FA acquired by using DTI with high $b$ values are useful for visualizing ischemic brain damage in apparently normal WM. In this study, patients with grade 2 or 3 PWML were excluded.

The results of our study are different compared to the aforementioned studies, which we do not see as a contradiction, but rather as an extension of the limited data on tissue integrity in ICAS. Our findings might well be explained by the characteristics of our patient population. We included only patients without any signs of prior cerebral infarction or TIA by careful individual patient history and clinical examination as well as by MRI. Therefore, they represent a 'strictly' asymptomatic population. Taken together, the findings of the present study and the previous data suggest a clinical continuum in ICAS with truly asymptomatic populations like the present one at the beginning and symptomatic patients at the end with disease duration as an important determinant. In addition, the investigation at a rather early stage of the disease and the observation of a relatively high prevalence of visible intracranial collaterals via the circle of Willis with 12 of 15 patients having either an anterior or posterior communicating artery ipsilateral to the stenosis or both might as well contribute to the present results. Accordingly, normal cerebral perfusion measurements in asymptomatic ICAS patients have been reported [14, 15].

\section{Limitations}

In the present study we investigated a rather small sample of 15 individuals resulting in reduced power of applied statistical tests.

\section{Conclusion}

Using quantitative DTI in asymptomatic patients with ICAS, we found no changes suggestive of white matter damage due to a unilateral high-grade carotid artery stenosis. This seems to reflect the benign nature of the disease in a particular subgroup of patients at a certain stage and can in part be attributed to the high prevalence of intracranial collaterals. Due to the small sample size, our findings have to be considered as preliminary and confirmation is needed.

\section{Disclosure Statement}

The authors report no conflict of interest.

References

$\checkmark 1$ Chambers BR, Norris JW: Outcome in patients with asymptomatic neck bruits. N Engl J Med 1986;315:860-865.

2 Hennerici $\mathrm{M}$, Hulsbomer $\mathrm{HB}$, Hefter $\mathrm{H}$, Lammerts D, Rautenberg W: Natural history of asymptomatic extracranial arterial disease. Results of a long-term prospective study. Brain 1987;110:777-791.

- 3 Reiff T, Stingele R, Eckstein HH, Fraedrich G, Jansen O, Mudra H, Mansmann U, Hacke W, Ringleb P: Stent-protected angioplasty in asymptomatic carotid artery stenosis vs. endarterectomy: SPACE2 - a three-arm randomised-controlled clinical trial. Int J Stroke 2009;4:294-299. 
4 Inoue T, Ogasawara K, Konno H, Ogawa A, Kabasawa H: Diffusion tensor imaging in patients with major cerebral artery occlusive disease. Neurol Med Chir (Tokyo) 2003;43: 421-425, discussion 426.

5 Shiraishi A, Hasegawa Y, Okada S, Kimura K, Sawada T, Mizusawa H, Minematsu K: Highly diffusion-sensitized tensor imaging of unilateral cerebral arterial occlusive disease. AJNR Am J Neuroradiol 2005;26:14981504.

6 Soinne L, Helenius J, Saimanen E, Salonen O, Lindsberg PJ, Kaste M, Tatlisumak T: Brain diffusion changes in carotid occlusive disease treated with endarterectomy. Neurology 2003;61:1061-1065.
7 Patankar T, Widjaja E, Chant H, McCollum C, Baldwin R, Jeffries S, Sutcliffe C, Burns A, Jackson A: Relationship of deep white matter hyperintensities and cerebral blood flow in severe carotid artery stenosis. Eur J Neurol 2006;13:10-16.

$\checkmark 8$ Beaulieu C: The basis of anisotropic water diffusion in the nervous system - a technical review. NMR Biomed 2002;15:435-455.

9 Le Bihan D: Looking into the functional architecture of the brain with diffusion MRI. Nat Rev Neurosci 2003;4:469-480.

10 Bogousslavsky J, Regli F: Borderzone infarctions distal to internal carotid artery occlusion: prognostic implications. Ann Neurol 1986;20:346-350.

11 Fazekas F, Chawluk JB, Alavi A, Hurtig HI, Zimmerman RA: MR signal abnormalities at $1.5 \mathrm{~T}$ in Alzheimer's dementia and normal aging. AJR Am J Roentgenol 1987;149:351356.
2 Haselgrove JC, Moore JR: Correction for distortion of echo-planar images used to calculate the apparent diffusion coefficient. Magn Reson Med 1996;36:960-964.

13 Basser PJ, Mattiello J, LeBihan D: Estimation of the effective self-diffusion tensor from the NMR spin echo. J Magn Reson B 1994;103: 247-254.

14 Chaves CJ, Staroselskaya I, Linfante I, Llinas R, Caplan LR, Warach S: Patterns of perfusion-weighted imaging in patients with carotid artery occlusive disease. Arch Neurol 2003;60:237-242.

15 Soinne L, Helenius J, Tatlisumak T, Saimanen E, Salonen O, Lindsberg PJ, Kaste M: Cerebral hemodynamics in asymptomatic and symptomatic patients with high-grade carotid stenosis undergoing carotid endarterectomy. Stroke 2003;34:1655-1661. 